\title{
STRUCTURE ET PROPRIÉTÉS DE SOLUTIONS SOLIDES BINAIRES DE NIOBIUM AVEC LE TITANE, LE MOLYBDẼNE ET LE ZIRCONIUM
}

\author{
par E. JOSSO
}

Société Imphy-Kuhlmann

\begin{abstract}
Résumé. - Les alliages étudiés sont des solutions solides de niobium contenant respectivement $10 \% \mathrm{Mo}, 20 \%$ Ti ou $4 \% \mathrm{Zr}$ (compositions pondérales). Seul le titane conserve presque intégralement l'exceptionnelle déformabilité du niobium ; le molybdène, et plus encore le zirconium, rétrécissent le domaine de forgeabilité. Les trois éléments exercent une importante action durcissante sur le niobium par effet de solution solide, mais ils favorisent peu son durcissement mécanique par écrouissage. Le molybdène et le zirconium relèvent d'au moins $100^{\circ} \mathrm{C}$ la température de recristallisation. Le titane, et surtout le zirconium, donnent lieu à une importante ségrégation interdendritique. L'alliage $\mathrm{Nb}-20 \mathrm{Ti}$ possède une médiocre tenue mécanique à chaud, mais fait montre d'un comportement quasi superplastique au-dessus de $1200^{\circ} \mathrm{C}$. En dépit de sa plus faible concentration atomique en élément d'addition, l'alliage $\mathrm{Nb}-4 \mathrm{Zr}$ présente une meilleure résistance à la traction que l'alliage Nb-10 Mo pour les températures supérieures à $800^{\circ} \mathrm{C}$.

Abstract. - Niobium solid solutions containing $10 \mathrm{wt} \% \mathrm{Mo}, 20 \mathrm{wt}$ Ti or $4 \mathrm{wt} \% \mathrm{Zr}$ have been studied. Only in the case of titanium is the exceptional workability of niobium almost entirely maintained, whereas its forgeability range is narrowed by molybdenum and especially by zirconium All three elements strongly harden niobium by solid solution strengthening but have little effect on its work hardening characteristics. Molybdenum and zirconium increase the recrystallization temperature by at least $100^{\circ} \mathrm{C}$. Titanium, and particulary zirconium, give rise to extensive interdendritic segregation. The $\mathrm{Nb}-20 \mathrm{Ti}$ alloy shows low mechanical strength at high temperatures with nearly superplastic behaviour above $1200^{\circ} \mathrm{C}$. In spite of its lower atomic solute content, the $\mathrm{Nb}-4 \mathrm{Zr}$ alloy shows a higher tensile strength than the Nb-10 Mo alloy above $800^{\circ} \mathrm{C}$.
\end{abstract}

Le niobium possède le privilège peu répandu de constituer la base d'alliages dont les applications potentielles s'échelonnent depuis les plus basses jusqu'aux plus élevées des températures industrielles. Associé au titane ou à l'étain, il s'est frayé une place de choix dans le domaine des supraconducteurs travaillant au voisinage du zéro absolu. Sa résistance chimique à l'état pur ou allié commence à lui ouvrir des débouchés aux températures voisines de l'ambiante. Enfin, ses alliages avec d'autres métaux à haut point de fusion figurent parmi ceux qui font montre de la meilleure tenue mécanique aux températures supérieures à $1100^{\circ} \mathrm{C}$, domaine où les alliages super-réfractaires à base de nickel ou de cobalt se révèlent défaillants. Depuis une quinzaine d'années notamment, on a vu naître un nombre important d'alliages de niobium possédant une haute résistance à chaud. Ceux d'entre eux qui ouvrent le plus d'espoirs contiennent généralement plusieurs éléments d'addition et sont parfois dopés par des interstitiels, tel le carbone.

La bibliographie se montre avare de renseignements motivant le choix de tel ou tel élément d'addition et explicitant le rôle particulier de chaque élément de dopage. Afin de contribuer à combler cette lacune, nous avons entrepris, dans le cadre d'un contrat DGRST, d'étudier l'action individuelle du molybdène, $\mathrm{du}$ zirconium et du titane qui figurent parmi les métaux les plus fréquemment associés au niobium et de déterminer l'influence du carbone, de l'oxygène et de l'azote sur les propriétés des alliages binaires ainsi formés.

Le présent exposé sera limité à la première partie de ce travail, c'est-à-dire à l'étude des alliages binaires exempts d'éléments de dopage. Le choix des compositions a été guidé par les considérations suivantes :

- l'alliage doit former une solution solide unique (d'après les diagrammes actuellement connus),

- la concentration en élément d'addition doit rester dans les limites des teneurs trouvées dans les alliages industriels.

Sur la base de ces critères, ont été retenues les compositions pondérales suivantes :

- alliage Nb-Mo à $10 \%$ Mo (en abrégé Nb10Mo); teneur en atomes : 9,7\%;

- alliage $\mathrm{Nb}-\mathrm{Ti}$ à $20 \% \mathrm{Ti}(\mathrm{Nb} 20 \mathrm{Ti})$; teneur en atomes : $32,7 \%$;

- alliage $\mathrm{Nb}-\mathrm{Zr}$ à $4 \% \mathrm{Zr}(\mathrm{Nb} 4 \mathrm{Zr})$; teneur en atomes : $4 \%$.

Matières premières. Techniques d'élaboration. La nomenclature et l'analyse des matières premières utilisées figurent dans le tableau I. Compte tenu des écarts de points de fusion des éléments constitutifs, 


\section{TABLEAU II}

Analyses chimiques

Chiffres donnés dans l'ordre : périphérie - mi-rayon - cœur. Quand il n'y a que deux chiffres, il s'agit de prélèvements moyens.

Nuance
Lingot
Elaboration

Lieu de prélèvement $\ldots .$.

Elém. d'alliage $\% \ldots . .$.

C ppm
$\mathrm{N}_{2}$ ppm
$\mathrm{O}_{2}$ ppm
Ta ppm
W ppm
Fe ppm
Si ppm
Mo ppm
Ti ppm
Zr ppm

Dureté Vickers (HV 30)

$$
\begin{aligned}
& \text { Nb10Mo } \\
& \text { F } 252 \\
& 1 \mathrm{EC}+2 \mathrm{BE}
\end{aligned}
$$

$\begin{array}{cc}\text { Tête } & \text { Pied } \\ - & - \\ 9,40 & 9,75 \\ 9,35 & 9,90 \\ 9,50 & 9,75\end{array}$

$20-22-22 \quad 33-26-22$

45-45-49 $\quad 66-56-35$

$45-50-83 \quad 13-41-57$

$35-35$

$<20-<20$

$<20-<20$

\section{$\mathrm{Nb20Ti}$ \\ L 771 \\ $3 \mathrm{EC}$}

$\mathrm{Nb} 4 \mathrm{Zr}$

F 254

$1 \mathrm{EC}+2 \mathrm{BE}$

\begin{tabular}{cc} 
Tête & Pied \\
\hline-10 & - \\
3,85 & 3,60 \\
3,85 & 3,60 \\
& 3,70
\end{tabular}

27-35-28 29-37-32

51-42-56 42-63-39

47-42-77 76-41-94

$60-50$

$<20-<20$

$<20-<20$

chaque alliage a subi trois fusions successives, afin d'améliorer l'homogénéité. Pour $\mathrm{Nb} 10 \mathrm{Mo}$ et $\mathrm{Nb} 4 \mathrm{Zr}$, la première fusion fut effectuée au four à électrode consommable et les deux suivantes au four à bombardement électronique dont le vide élevé et les possibilités de surchauffe favorisent l'élimination des impuretés. Ce processus s'est révélé impraticable avec l'alliage $\mathrm{Nb20Ti}$ en raison de la tension de vapeur du titane qui entraînait la volatilisation quasi complète de cet élément pendant la fusion par bombardement

\begin{tabular}{|c|c|c|c|c|c|c|}
\hline Eléments & Repère F 159 & $\begin{array}{c}\text { Niobium } \\
\text { Repère F } 196\end{array}$ & Repère F 250 & Molybdène & $\begin{array}{c}\text { Titane } \\
\text { (Epingle } \\
\text { Van Arkel) }\end{array}$ & $\begin{array}{c}\text { Zirconium } \\
\text { (Epingle } \\
\text { Van Arkel) }\end{array}$ \\
\hline$\overline{\mathrm{C}}$ & $26-74$ & $25-46$ & 38 & $\overline{14}$ & $\overline{10}$ & 62 \\
\hline $\begin{array}{l}\mathrm{N}_{2} \\
\mathrm{O}_{2}\end{array}$ & $56-119$ & 63 & 61 & 14 & 20 & 150 \\
\hline Ta & $25-35$ & $35-45$ & 35 & & & \\
\hline W & $<20$ & $<20$ & $<20$ & & & $<30$ \\
\hline $\mathrm{Fe}$ & $<20$ & $20-23$ & $<20$ & 28 & 20 & 20 \\
\hline $\mathrm{Si}$ & $16-19$ & $16-42$ & 17 & 37 & 50 & $<30$ \\
\hline Mo & $<20$ & $<20$ & $<20$ & & & $<30$ \\
\hline $\mathrm{Ti}$ & $<20$ & $<20$ & $<20$ & & & $<10$ \\
\hline $\mathrm{Zr}$ & $<65$ & $<65$ & $<65$ & & 500 & \\
\hline $\mathrm{Sn}$ & $<20$ & $<20$ & $<20$ & & 10 & $<10$ \\
\hline $\mathrm{Ni}$ & $<10$ & $<10$ & $<10$ & & 1 & $<20$ \\
\hline $\mathrm{Al}$ & $<50$ & $<50$ & $<50$ & 5 & 50 & 16 \\
\hline $\mathrm{Cu}$ & $<30$ & $<30$ & $<30$ & & 3 & $<10$ \\
\hline
\end{tabular}

\section{TABLEAU I}

Composition des matières premières (ppm)

électronique. Pour cette composition, on a eu recours à une triple fusion à l'électrode consommable. Les lingots obtenus avaient un diamètre de $80 \mathrm{~mm}$ (ramené à 70-77 $\mathrm{mm}$ par écroûtage) et pesaient environ $7 \mathrm{~kg}$. Leurs analyses chimiques sont rassemblées dans le tableau II qui donne les compositions en tête et en pied pour des prélèvements effectués en périphérie, à mi-rayon et à cœur. Le problème du rendement des additions ( $\mathrm{Mo}, \mathrm{Ti}, \mathrm{Zr}$ ) peut être considéré comme maîtrisé, puisque les teneurs obtenues sont à 
peu de chose près celles visées. A l'échelle macroscopique, l'hétérogénéité de répartition de ces éléments d'alliage reste faible. On verra par la suite qu'il n'en va pas toujours de même à l'échelle microscopique. En ce qui concerne les impuretés métalloïdiques, il importe de souligner que les nuances Nb10Mo et $\mathrm{Nb} 4 \mathrm{Zr}$ sont plus pauvres en carbone et en oxygène (et aussi en azote, mais dans une moindre mesure) que la nuance Nb20Ti. Cette différence concrétise l'efficacité de la purification par bombardement électronique.

Forgeage. - Pour éviter une consommation exagérée de métal que ne permettait pas d'envisager le faible volume des lingots, le domaine de forgeabilité a été déterminé au moyen d'essais de refoulement sur pastilles de $20 \mathrm{~mm}$ de diamètre et $13 \mathrm{~mm}$ d'épaisseur.

Comme pour le niobium pur, la plage de forgeabilité de l'alliage $\mathrm{Nb20Ti}$ s'avère très vaste, puisqu'elle s'étend depuis $300^{\circ} \mathrm{C}$ jusqu'aux températures très élevées. Bien que moins malléable, la nuance $\mathrm{Nb} 10 \mathrm{Mo}$ se forge correctement entre 800 et $1100^{\circ} \mathrm{C}$. L'alliage à $4 \% \mathrm{Zr}$ se révèle le plus difficile à forger. On arrive néanmoins à le corroyer avec des gerçures mais sans criques profondes au voisinage de $1200^{\circ} \mathrm{C}$.

Une addition de $4 \% \mathrm{Zr}$ suffit donc à faire perdre en majeure partie l'exceptionnelle aptitude à la déformation du niobium, sans toutefois supprimer la possibilité de corroyage.

Structure. - Le lingot d'alliage Nb10Mo est caractérisé par une cristallisation colonnaire extrêmement grossière (Fig. 1) qui contraste avec la cristallisation fine de l'alliage $\mathrm{Nb} 4 \mathrm{Zr}$ élaboré suivant un processus identique (Fig. 2). L'effet bien connu du titane sur l'affinage du grain est illustré par la figure 3 .

A l'état brut de coulée, les nuances Nb10Mo et Nb20Ti se présentent comme des solutions solides homogènes, conformément à leur diagramme d'équilibre. Au contraire, dans la microstructure de l'alliage $\mathrm{Nb} 4 \mathrm{Zr}$, on dépiste une seconde phase qui se présente

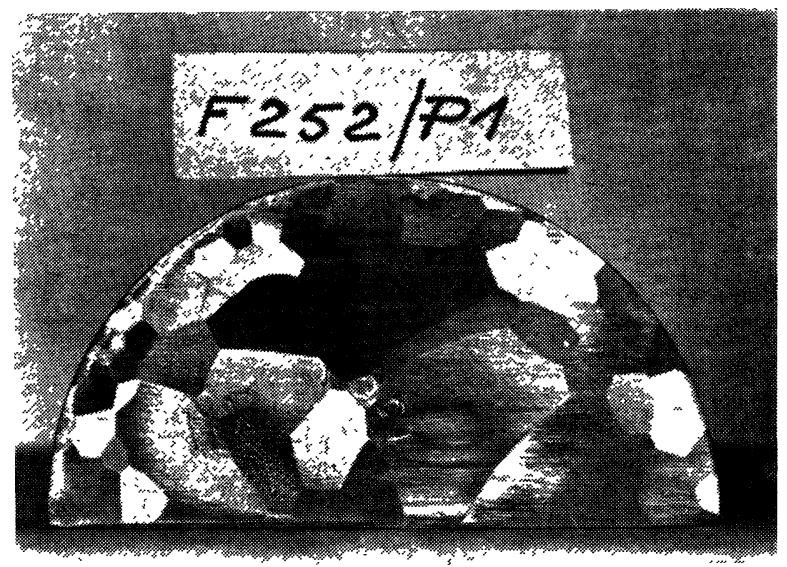

FIG. 1. - Alliage Nb10Mo élaboré par bombardement électronique. Structure macrographique à l'état brut de coulée. $G=1$.

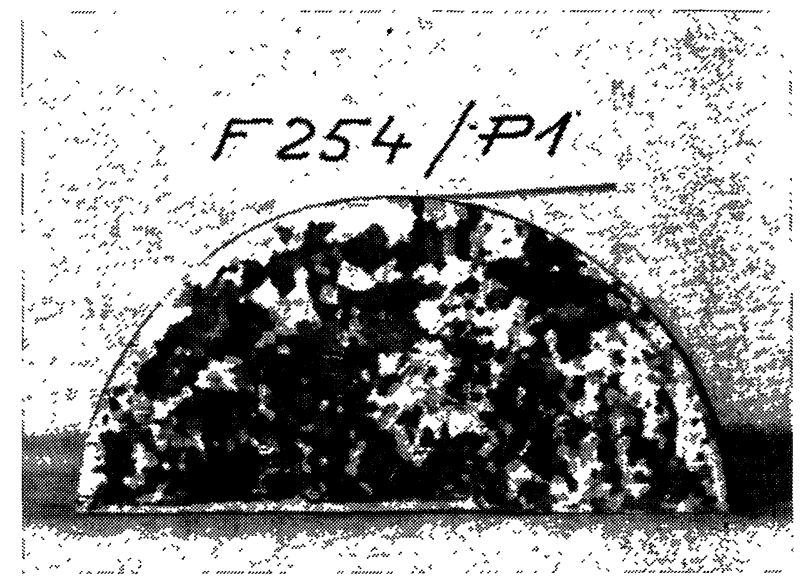

FIG. 2. - Alliage Nb4Zr élaboré par bombardement électronique. Structure macrographique à l'état brut de coulée. $G=1$.

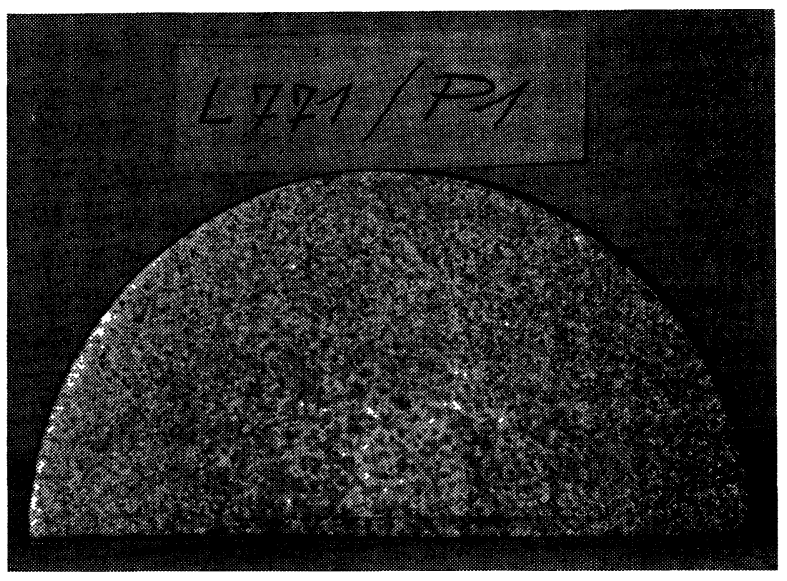

Fig. 3. - Alliage Nb20Ti élaboré au four à électrode consommable. Structure macrographique à l'état brut de coulée. $G=1$.

sous forme de lignes continues ou ponctuées à parcours sinueux (Fig. 4). L'examen microfractographique ne décèle aucun constituant à la surface des cassures de Nb10Mo et Nb20Ti, alors qu'il met en évidence la

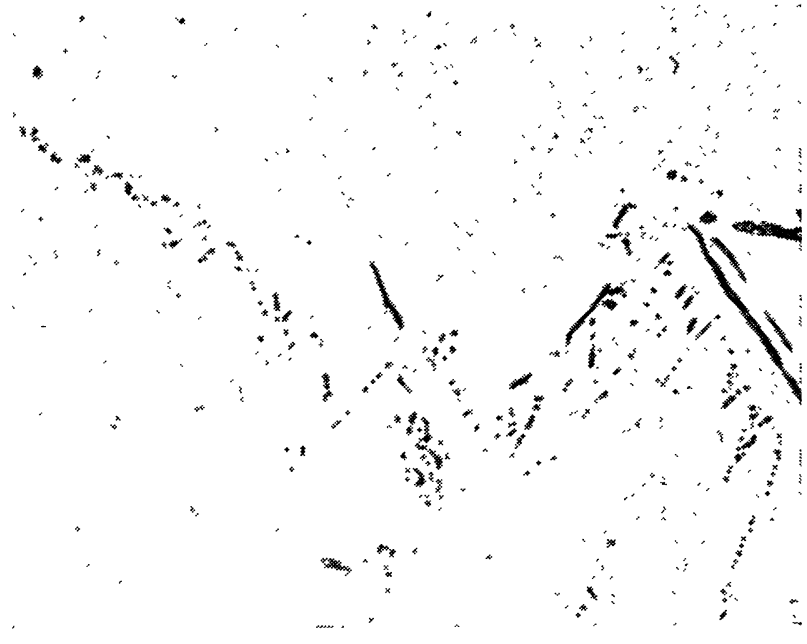

FIg. 4. - Alliage Nb4Zr. Morphologie du constituant parasite. $G=500$ 
phase parasite de $\mathrm{Nb} 4 \mathrm{Zr}$ sous forme de lamelles plus ou moins fragmentées par le choc de rupture (Fig. 5), que la diffraction électronique identifie à l'oxyde de zirconium $\mathrm{ZrO}_{2}$. Ainsi, malgré une méthode raffinée d'élaboration, et en dépit de sa faible teneur globale en oxygène, l'alliage a emprisonné des films de zircone, résidus probables de « peaux »d'oxydation superficielles. Selon toute vraisemblance, ces films sont au moins partiellement responsables des gerçures observées à la partie périphérique des pièces forgées en $\mathrm{Nb} 4 \mathrm{Zr}$.

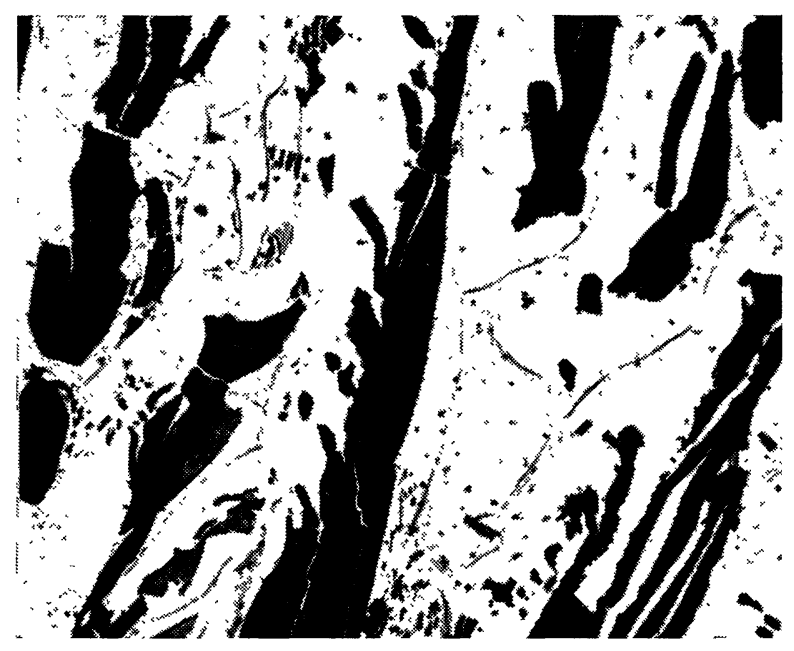

FIG. 5. - Alliage Nb4Zr. Réplique avec extraction. Faciès du constituant parasite. $G=4000$.

Homogénéité. - L'alliage Nb20Ti soupçonné d'une importante hétérogénéité dendritique en raison de l'écart supérieur à $200^{\circ} \mathrm{C}$ entre les températures de son liquidus et de son solidus a été soumis à l'exploration microchimique au moyen de la sonde de Castaing. Les mêmes déterminations ont été effectuées sur les alliages $\mathrm{Nb} 10 \mathrm{Mo}$ et $\mathrm{Nb} 4 \mathrm{Zr}$ à titre de comparaison. Les échantillons avaient subi un corroyage à chaud modéré, très insuffisant pour assurer l'homogénéisation, suivi d'écrouissage à froid. Ils ont été examinés à l'état écroui ainsi qu'à l'état recuit à $1200^{\circ} \mathrm{C}$ (durée : 1 heure) sous vide. Deux régions différentes de chaque spécimen ont été explorées. Les résultats sont consignés dans le tableau III où l'hétérogénéité à l'échelle microscopique

\section{TABLEAU III}

Indices de ségrégation du $\mathrm{Nb}$ dans ses alliages avec Mo, $\mathrm{Ti}$ ou $\mathrm{Zr}$

\begin{tabular}{lcccc} 
& & \multicolumn{3}{c}{ Indice de ségrégation } \\
Nuance & Etat & 1 & 2 & \\
Zone & Zone & Moyenne \\
Nb10Mo & - & - & - & - \\
& Ecroui & 1,11 & 1,10 & 1,10 \\
$\mathrm{Nb} 20 \mathrm{Ti}$ & Recuit 1 h à $1200^{\circ} \mathrm{C}$ & 1,09 & 1,06 & 1,07 \\
& Ecroui & 1,43 & 1,23 & 1,33 \\
$\mathrm{Nb} 4 \mathrm{Zr}$ & Recuit 1 h à $1200^{\circ} \mathrm{C}$ & 1,33 & 1,32 & 1,32 \\
& Ecroui & 1,57 & 1,54 & 1,55 \\
& Recuit 1 h à $1200^{\circ} \mathrm{C}$ & 1,16 & 1,19 & 1,18
\end{tabular}

est chiffrée arbitrairement par "l'indice de ségrégation", rapport entre les concentrations maximale et minimale du niobium.

$\mathrm{Si}$, répondant aux prévisions, l'indice de ségrégation atteint effectivement une valeur élevée pour Nb20Ti, c'est en définitive l'alliage $\mathrm{Nb} 4 \mathrm{Zr}$ qui fait montre de la plus forte hétérogénéité dendritique, du moins avant recuit. La ségrégation mineure reste faible pour Nb10Mo.

Le recuit d'une heure à $1200^{\circ} \mathrm{C}$ s'avère insuffisant dans le cas de Nb10Mo et Nb20Ti alors qu'il provoque déjà un effet d'homogénéisation remarquable dans $\mathrm{Nb} 4 \mathrm{Zr}$.

Les familles d'alliages $\mathrm{NbTi}$ et $\mathrm{NbZr}$ figurant parmi les meilleurs des matériaux supraconducteurs de seconde espèce, on est en droit de se demander dans quelle mesure leur propension à la ségrégation interdendritique peut favoriser leur supraconductivité. En effet, il existe une différence de paramètre cristallin entre deux zones adjacentes, l'une riche l'autre pauvre en $\mathrm{Ti}$ ou en $\mathrm{Zr}$. Il y a donc perturbation du réseau et accumulation corrélative de défauts à leur interface. Comme déjà signalé au récent colloque d'Aussois [1], ces défauts seraient susceptibles de créer des centres de piégeage du flux suivant un mécanisme d'effet de surface " interne " rappelant les effets de joints de grains décrits par J. Maldy [2]. Ces éventuels centres de piégeage seraient extrêmement nombreux car les distances entre zones riches et pauvres sont petites vis-à-vis des dimensions des grains.

Ecrouissage - Recristallisation. - Pris à l'état brut de forgeage par refoulement, état mal défini mais déjà partiellement écroui, les trois alliages étudiés se comportent de façon assez similaire lors des déformations à l'ambiante : ils durcissent peu quand on les soumet à un écrouissage complémentaire par laminage, même lorsqu'ils subissent d'importantes réductions de section (Fig. 6). Paradoxalement, la dureté Vickers des nuances Nb10Mo et Nb20Ti commence même par décroître aux faibles taux de déformation, tandis que celle de $\mathrm{Nb} 4 \mathrm{Zr}$ reste stationnaire.

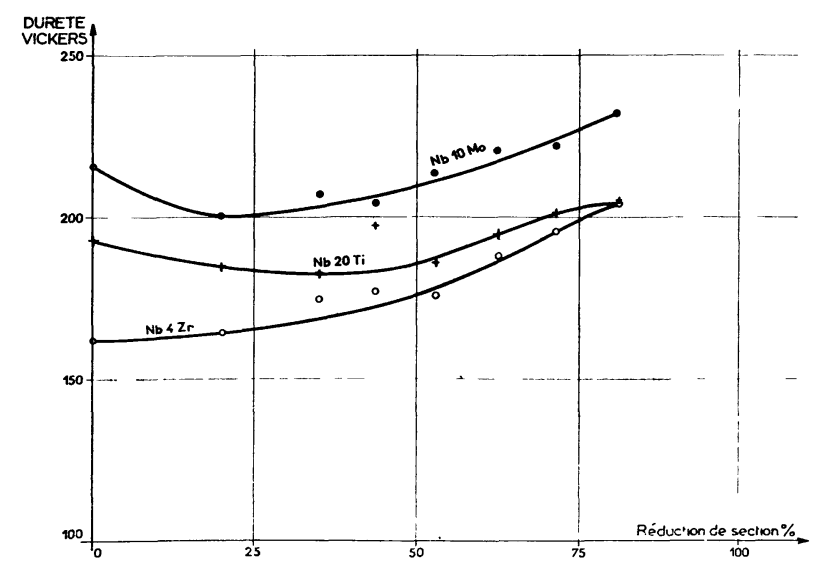

FIg. 6. - Evolution de la dureté en fonction du taux d'écrouissage. 
En revanche, l'effet d'adoucissement provoqué par un recuit d'une heure à température comprise entre 700 et $1200^{\circ} \mathrm{C}$ sur échantillons fortement écrouis (réduction de section supérieure à $80 \%$ ) se manifeste de manière fort différente suivant l'élément d'addition considéré, comme le montre la figure 7. La variation de dureté de $\mathrm{Nb} 10 \mathrm{Mo}$ reste progressive et régulière.

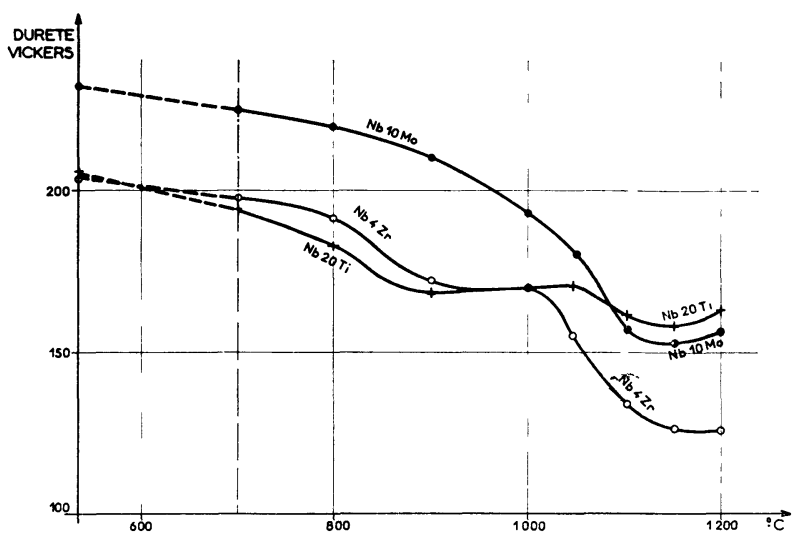

FIG. 7. - Evolution de la dureté en fonction de la température de recuit (durée : 1 heure).

Le début de la recristallisation entre 1000 et $1050^{\circ} \mathrm{C}$ ne se signale par aucune singularité et la dureté continue à baisser pendant que la recristallisation se développe et que le grain grossit. Le léger relèvement de dureté entre 1150 et $1200^{\circ} \mathrm{C}$, observable aussi sur les autres nuances, doit vraisemblablement être porté au compte d'un début de contamination par un vide insuffisamment propre, suivant un mécanisme déjà décrit dans une publication antérieure [4].

La dureté de Nb20Ti commence par décroître de manière importante puisqu'elle tombe de 205 à $168 \mathrm{~kg} / \mathrm{mm}^{2}$ entre l'ambiante et $900{ }^{\circ} \mathrm{C}$, température de début de recristallisation décelée par examen micrographique. Au-delà de ce seuil, elle ne varie plus que très lentement et reste encore égale à $160 \mathrm{~kg} / \mathrm{mm}^{2}$ après recuit à $1150^{\circ} \mathrm{C}$. L'essentiel de l'adoucissement s'est donc produit pendant la période de restauration. La recristallisation et le grossissement $\mathrm{du}$ grain n'exercent qu'une action minime sur la dureté.

Le comportement de l'alliage $\mathrm{Nb} 4 \mathrm{Zr}$ s'avère plus complexe. Il y a séparation nette de la période de restauration qui s'achève vers $900^{\circ} \mathrm{C}$ et de la phase de recristallisation qui débute vers $1000^{\circ} \mathrm{C}$, par un palier étalé sur une centaine de degrés pendant lequel la dureté reste pratiquement inchangée.

Propriétés mécaniques de traction à chaud. Les caractéristiques de traction ont été mesurées sur microéprouvettes Chevenard (diamètre : $2 \mathrm{~mm}$, longueur utile : $10 \mathrm{~mm}$ ) avec une vitesse de déplacement de la traverse égale à $0,8 \mathrm{~mm} / \mathrm{mn}(8 \% / \mathrm{mn})$. Chaque éprouvette était maintenue $15 \mathrm{mn}$ à la température d'essai avant mise en charge.
Les éprouvettes ont été usinées dans des ébauches préalablement recuites $1 \mathrm{~h}$ à $1000^{\circ} \mathrm{C}$ sous vide. Ce traitement provoque la recristallisation quasi complète de la nuance $\mathrm{Nb20Ti}$, mais ne fait qu'amorcer la recristallisation des deux autres nuances.

Les figures 8,9 et 10 illustrent respectivement l'évolution de la limite élastique à $0,2 \%$, de la charge de rupture et de l'allongement en fonction de la température de traction. Il existe une grande similitude entre les figures 8 et 9 car la charge de rupture reste voisine de la limite élastique. En conséquence, nous nous bornerons à analyser les variations de la charge de rupture (Fig. 9) et de l'allongement (Fig. 10).

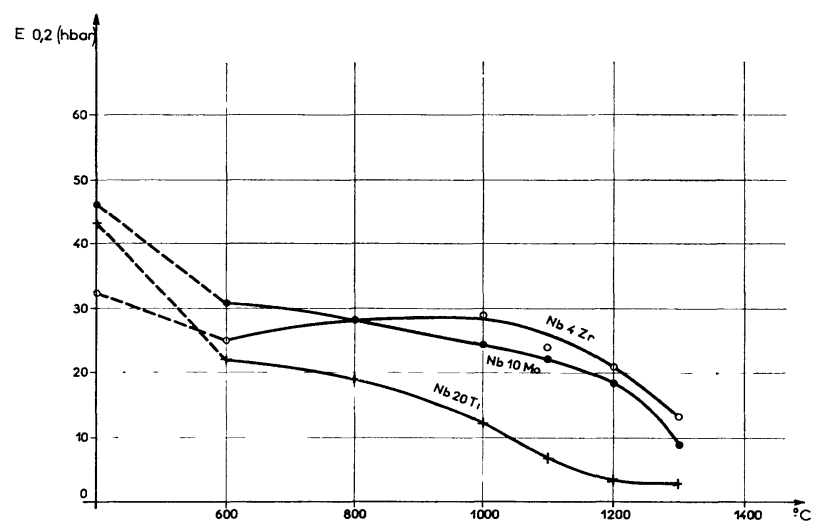

FIg. 8. - Evolution de la limite élastique à $0,2 \%$ en fonction de la température de recuit (durée : 1 heure).

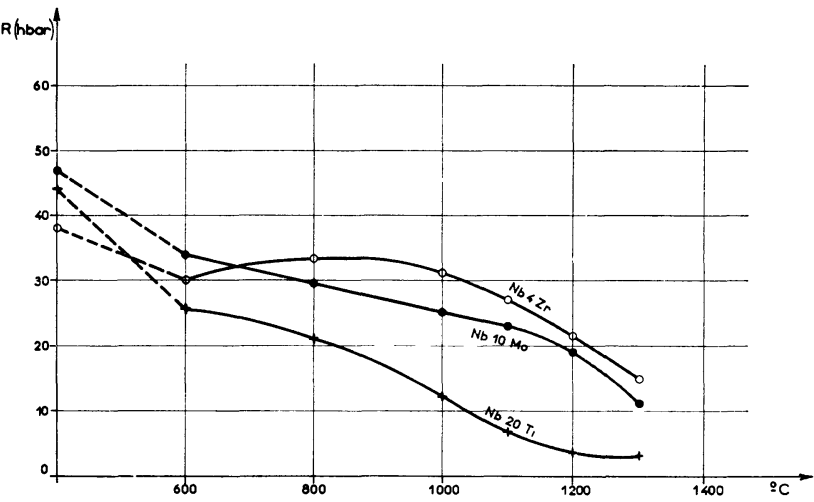

Fig. 9. - Evolution de la charge de rupture en fonction de la température de recuit (durée : 1 heure).

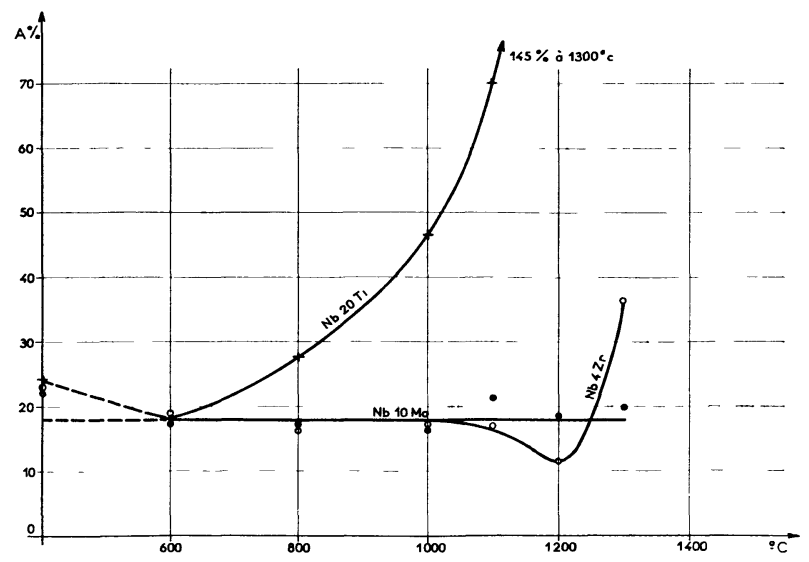

FIG. 10. - Evolution de l'allongement de rupture en fonction de la température de recuit (durée : 1 heure). 
Charge de rupture (Fig. 9). - A la température ambiante, les nuances $\mathrm{Nb} 10 \mathrm{Mo}$ et $\mathrm{Nb} 20 \mathrm{Ti}$ possèdent à peu près la même résistance mécanique de l'ordre de 45 hbar, nettement supérieure à celle de $\mathrm{Nb} 4 \mathrm{Zr}$ qui ne dépasse pas 38 hbar. Même à ce niveau, l'effet durcissant du $\mathrm{Zr}$ doit tout de même être considéré comme important puisque la charge de rupture du niobium de bonne pureté se situe vers $20 \mathrm{hbar}$. La tenue mécanique de $\mathrm{Nb10Mo}$ décroît régulièrement lorsque la température s'élève. Il en est de même pour Nb20Ti, la chute étant toutefois beaucoup plus rapide. Pour fixer les idées, la charge de rupture de Nb10Mo dépasse encore légèrement 11 hbar à $1300^{\circ} \mathrm{C}$ alors que celle de Nb20Ti n'atteint même pas 3 hbar.

Ici encore, l'alliage au zirconium se singularise. Après une chute de résistance entre l'ambiante et $600{ }^{\circ} \mathrm{C}$, il voit sa charge de rupture remonter, passer par un maximum égal à 33 hbar vers $900^{\circ} \mathrm{C}$ pour descendre ensuite lentement jusque vers 15 hbar à $1300^{\circ} \mathrm{C}$. Inférieur à l'alliage Nb10Mo entre l'ambiante et $700{ }^{\circ} \mathrm{C}$, l'alliage $\mathrm{Nb} 4 \mathrm{Zr}$ devient plus tenace que lui pour toutes les températures supérieures ou égales à $800^{\circ} \mathrm{C}$.

D'après le diagramme d'équilibre de Rogers et Atkins [3], la teneur de $4 \% \mathrm{Zr}$ n'est pas très éloignée de la frontière des alliages diphasés. Compte tenu de l'hétérogénéité dendritique signalée précédemment, il est certain que cette frontière est franchie dans les zones enrichies en $\mathrm{Zr}$. Il paraissait donc légitime d'attribuer le raidissement de l'alliage entre 600 et $1000^{\circ} \mathrm{C}$ à la précipitation de la seconde phase prévue par le diagramme d'état. Toutefois, jusqu'à présent, cette hypothèse n'a pas pu être confirmée par les examens radiocristallographiques de la structure.

Allongements (Fig. 10). - Une assez grande dispersion, éventuellement liée au grain insuffisamment affiné par un corroyage trop modéré, se manifeste pour les alliages $\mathrm{Nb} 10 \mathrm{Mo}$ et $\mathrm{Nb} 4 \mathrm{Zr}$. Compte tenu de l'incertitude qui en résulte, on peut considérer l'allongement de rupture de $\mathrm{Nb} 10 \mathrm{Mo}$ comme pratiquement constant entre l'ambiante et $1300^{\circ} \mathrm{C}$. Sa grandeur oscille entre 16 et $22 \%$.

Il en va de même pour $\mathrm{Nb} 4 \mathrm{Zr}$ jusqu'à $1100^{\circ} \mathrm{C}$; à $1200{ }^{\circ} \mathrm{C}$ son allongement tombe à $11 \%$ pour remonter au-delà de $35 \%$ à $1300 \% \mathrm{C}$.

Le comportement de $\mathrm{Nb} 20 \mathrm{Ti}$ se révèle bien différent. Doté d'un allongement proche de $25 \%$ à l'ambiante, il présente un trou de ductilité relatif vers $600^{\circ} \mathrm{C}$, température au-delà de laquelle l'allongement se met à croître très vite avec la température pour atteindre $145 \%$ et $1300{ }^{\circ} \mathrm{C}$. Entre 1200 et $1300{ }^{\circ} \mathrm{C}$ l'allongement dépasse $100 \%$ et, à certains égards, le comportement de l'alliage au titane rappelle celui des matériaux superplastiques.

Conclusion. - De cette revue des propriétés des alliages $\mathrm{Nb} 10 \mathrm{Mo}, \mathrm{Nb} 20 \mathrm{Ti}, \mathrm{Nb} 4 \mathrm{Zr}$ préparés avec des matières premières exceptionnelles se dégagent les enseignements suivants :

- Parmi les trois éléments d'additions considérés, seul le titane permet de conserver presque intégralement l'exceptionnelle aptitude à la déformation du niobium. Le molybdène, et plus encore le zirconium restreignent considérablement les possibilités de mise en forme et rétrécissent le domaine de forgeabilité. L'absence de nocivité du Ti ne peut pas être attribuée exclusivement à son effet d'affinage du grain, puisque le zirconium qui exerce une action similaire, encore que moins efficace, se révèle pourtant plus nuisible à la forgeabilité que le molybdène dont l'influence sur la cristallisation de première consolidation est considérée comme nulle.

- En contre partie, les additions de Mo et $\mathrm{Zr}$ relèvent d'au moins $100^{\circ} \mathrm{C}$ la température de recristallisation, ce qui n'est pas sans intérêt pour les applications mécaniques à haute température.

- Les alliages $\mathrm{Nb} 20 \mathrm{Ti}$ et $\mathrm{Nb} 4 \mathrm{Zr}$, qui appartiennent à deux familles réputées pour leurs propriétés supraconductrices, donnent lieu à une importante ségrégation dendritique. On en a déduit un mécanisme possible de piégeage des lignes de flux.

- Les trois éléments d'addition exercent par euxmêmes une importante action durcissante, mais ne favorisent pas le durcissement mécanique par écrouissage. En raison de sa plus faible concentration atomique, l'alliage $\mathrm{Nb} 4 \mathrm{Zr}$ possède, à l'ambiante, des propriétés mécaniques inférieures à celles des deux autres nuances. Malgré ce handicap, sa tenue devient la meilleure dès que la température dépasse $800^{\circ} \mathrm{C}$.

Le titane se révèle nuisible à la résistance mécanique à haute température. Son action défavorable sur la charge de rupture à chaud était déjà connue, mais, sauf erreur, le comportement presque "superplastique » de l'alliage Nb20Ti aux températures égales ou supérieures à $1200^{\circ} \mathrm{C}$ n'avait pas encore été signalé. Il était d'ailleurs imprévisible d'après les théories admises puisqu'il ne paraît lié ni à la présence d'une seconde phase ni à une structure spécialement fine.

Pour intéressantes que soient les caractéristiques mécaniques de l'alliage $\mathrm{Nb} 4 \mathrm{Zr}$ à $1300^{\circ} \mathrm{C}$, elles restent encore inférieures à celles de certains alliages industriels plus complexes. La question que nous allons nous efforcer d'éclaircir dans la suite de notre étude consiste à savoir s'il est nécessaire de recourir à des alliages ternaires, voire quaternaires ou si une addition judicieusement dosée d'éléments interstitiels suffirait à remonter la tenue mécanique des alliages binaires au niveau des produits industriels actuellement utilisés.

Remerciements. - Nous tenons à exprimer nos remerciements à la DGRST pour le soutien matériel qu'elle a apporté à cette étude et pour son autorisation de publier les résultats de ces travaux. 


\section{Bibliographie}

[1] Josso (E.), Colloque sur les Matériaux Supraconducteurs (tables rondes ( Piégeage ) et (" Matériaux pour hauts champs )), 73, Aussois, 10-12 mars 1969.

[2] Maldy (J.), Colloque sur les Matériaux Supraconduc- teurs (table ronde ( Piégeage )), 73, Aussois, 10-12 mars 1969.

[3] Rogers (B. A.), Atkins (D. F.), Trans. AIME, 1955, 203, 1034-1041.

[4] Josso (E.) et Palmier-Gerber (M.), Mém. Scient. Rev. Métallurgie, 1965, 62, 479-484. 